\title{
Influence of sex and gender on the management of late-stage knee osteoarthritis
}

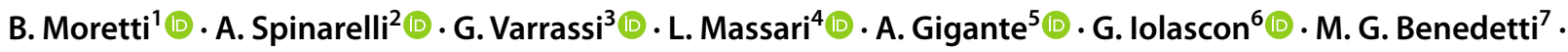 \\ A. M. Moretti ${ }^{8}$
}

Received: 19 April 2021 / Accepted: 25 July 2021 / Published online: 7 August 2021

(c) The Author(s) 2021

\begin{abstract}
Purpose The exact nature of sex and gender differences in knee osteoarthritis (OA) among patient candidates for total knee arthroplasty (TKA) remains unclear and requires better elucidation to guide clinical practice. The purpose of this investigation was to survey physician practices and perceptions about the influence of sex and gender on knee OA presentation, care, and outcomes after TKA.

Methods The survey questions were elaborated by a multidisciplinary scientific board composed of 1 pain specialist, 4 orthopedic specialists, 2 physiatrists, and 1 expert in gender medicine. The survey included 5 demographic questions and 20 topic questions. Eligible physician respondents were those who treat patients during all phases of care (pain specialists, orthopedic specialists, and physiatrists). All survey responses were anonymized and handled via remote dispersed geographic participation.

Results Fifty-six physicians ( $71 \%$ male) accepted the invitation to complete the survey. In general, healthcare professionals expressed that women presented worse symptomology, higher pain intensity, and lower pain tolerance and necessitated a different pharmacological approach compared to men. Pain and orthopedic specialists were more likely to indicate sex and gender differences in knee OA than physiatrists. Physicians expressed that the absence of sex and gender-specific instruments and indications is an important limitation on available studies.

Conclusions Healthcare professionals perceive multiple sex and gender-related differences in patients with knee OA, especially in the pre- and perioperative phases of TKA. Sex and gender bias sensitivity training for physicians can potentially improve the objectivity of care for knee OA among TKA candidates.
\end{abstract}

Keywords Gender · Osteoarthritis · Pain $\cdot$ Sex bias · Total knee arthroplasty

A. Spinarelli

antoniospinarelli@gmail.com

1 Department of Medical Sciences of Basis, Neurosciences and Organs of Sense, General Hospital, Faculty of Medicine and Surgery, University of Study of Bari, Bari, Italy

2 Department of Orthopedic and Trauma Unit, AOU Policlinico Consorziale Hospital, P.zza G. Cesare 11, 70124 Bari, BA, Italy

3 Paolo Procacci Foundation, Rome, Italy

4 Department of Biomedical and Specialty Surgical Sciences, University of Ferrara, Ferrara, Italy
5 Clinical Orthopedics, Department of Clinical and Molecular Science, Università Politecnica Delle Marche and Ospedali Riuniti Ancona, Ancona, Italy

6 Department of Medical and Surgical Specialties and Dentistry, University of Campania "Luigi Vanvitelli", Naples, Italy

7 Physical Medicine and Rehabilitation Unit, IRCCS-Istituto Ortopedico Rizzoli, Bologna, Italy

8 Italian Group for Health and Gender, Bari, Italy 


\section{Background}

Osteoarthritis (OA) is the leading cause of functional disability and reduced quality of life among adults in developed countries. OA of the knee accounts for more than $80 \%$ of the total disease burden [1] with an annual incidence of 181.2 per 100,000 population worldwide, representing a $8.2 \%$ increase from 1990 [2]. In a population-based study of older adults in Italy, $74 \%$ of individuals met the American College of Rheumatology clinical criteria for knee OA and $1 / 3 \mathrm{had}$ some form of symptomatic peripheral OA [3].

The prevalence of knee OA continues to grow alongside increases in its risk factors such as age and obesity [4].

Nonetheless, the exact nature of sex and gender differences in knee OA and especially in-patient candidates for TKA remains unclear and requires better elucidation before it can influence clinical practice for the purpose of improving patient care.

Sex and gender interact in complex ways to affect health outcomes [5-7]; sex refers to biological differences between men and women that involves physical and physiological characteristics, including chromosomes, gene expression, hormone levels, and reproductive/sexual anatomy, that may play a role in the pathogenesis of disease and response to treatment. Gender refers to socially constructed characteristics such as norms, roles, and relations influenced by society and culture at the time. People develop their gender roles in response to the environment in which they grow up and live, in response to the upbringing they receive at home and at school, depending on family and peer group interactions and expectations and according to trends and fashions in the media. In addition, political affiliation, social status, and economic availability can modify gender roles, that in some societies are more rigid than those in others Gender contribute to differences in vulnerabilities and susceptibilities to illness, can influence experiences of crises and emergency situations, health behaviors and how illness is experienced; gender influences also access to healthcare, uptake of health services, and globally health outcomes [6].

Female sex is a well-established risk factor for knee OA in older adults [8-13]. Moreover, risk factors such as obesity are stronger predictors among women than men due to inflammatory and mechanical disease drivers [14]. Previous studies indicate that women over the age of 50 have a higher prevalence of knee OA and experience greater functional disability compared to men of the same age, but the reasons for this difference are poorly understood $[15,16]$. Painful knee $\mathrm{OA}$ is also an independent predictor of mortality in middle-aged women [17].

Among available treatments, total knee arthroplasty (TKA) is a safe and cost-effective option that alleviates both pain and functional limitation related to end-stage knee OA [18-20]. Emerging data suggest that sex and gender-related differences in knee OA may also exist surrounding treatment. Despite a predilection for female sex, fewer women with symptomatic OA report discussing possible treatment with a physician than men, and women undergoing TKA tend to be treated at a later stage of disease associated with higher severity and functional disability than men [21, 22]. A more recent investigation reported that women have significantly higher healthcare utilization related to knee OA in the 12 months preceding TKA than men [23]. In terms of symptoms, a body of literature has established important sex and gender differences in the experience of pain, both related to physiological or biochemical differences [24] and psychological coping strategies [25], as well as sex differences in responses to pharmacological treatment [26]. With regard to TKA outcomes, one study reported that female sex was an independent risk factor for nonhome discharge but a protective factor against post-procedural complications [27].

Here, we performed a survey of healthcare professionals in Italy who attend candidates for TKA during all phases of care (pain specialists, orthopedic specialists, and physiatrists) in order to inform physician perceptions about the influence of sex and gender on knee OA presentation and management.

\section{Methods}

\section{Study design}

This study was a survey of physicians who treat patient candidates for TKA during all phases of care (preoperative, perioperative, and postoperative/rehabilitative) in Italy. Survey questions were designed to focus on the influence of sex and gender on knee OA presentation, care, and outcomes of TKA. Questions were elaborated by a multidisciplinary scientific board composed of 1 pain specialist, 4 orthopedic specialists, 2 physiatrists, and 1 expert in gender medicine. The survey was active for a period of 8 weeks (July $6,2020-$ September 1,2020 ) and communicated to a panel of physicians via e-mail using a password-protected web link. All survey responses were anonymized and handled via remote dispersed geographic participation.

\section{Survey}

The survey included 5 demographic questions and 21 survey questions about common practices and perceptions related to the influence of sex or gender on symptomology and pain severity, pharmacological therapy, postoperative outcomes, rehabilitation strategy, and patient satisfaction (see "Appendix" for list of survey questions). Question responses 
were multiple-choice and either single response or multiple response depending on the nature of the question. Responses were tabulated descriptively (number and percentage) for the overall group and by physician specialty. For questions that allowed open-ended response justifications, comment responses were analyzed thematically.

\section{Results}

\section{Respondent characteristics}

The survey was sent to 122 healthcare professionals in Italy; of these, 56 (46\%) accepted the invitation to participate and six respondents provided incomplete survey responses. Professional categories included anesthesiologists and pain specialists (9 respondents), physiatrists (17 respondents), orthopedic specialists (23 respondents), and the scientific board (7 respondents). Most respondents were male (71\%), aged between $41-60$ years (53\%), worked in university settings (57\%) and were geographically located in the south of Italy (46\%). Twenty-seven percent of respondents saw up to 10 patients with knee OA or knee replacement per month, $37 \%$ saw between 11 and 20 patients, and $36 \%$ saw more than 20 patients per month.

\section{Preoperative symptomatology and pain severity}

Sixty-seven percent of healthcare professionals disagreed with the assertion that male patients exhibit a more severe symptomatology than women upon first presentation (Q1), whereas $29 \%$ agreed with this assertion, and $4 \%$ were in total agreement. In contrast, $74 \%$ of respondents agreed or totally agreed that, among candidates with equivalent clinical and functional characteristics, women presented with poorer subjective impressions of functional limitation than men (Q2).

When characterizing preoperative pain severity among candidates for knee replacement (Q3), the overall impression was that pain is less tolerated by female patients (60\%), whereas 38\% expressed that pain is more intense in female patients, and an equivalent proportion expressed that pain intensity is absolutely independent of sex (Fig. 1). Forty percent of respondents indicated that there was no bearing of sex on subsequent pharmacological intervention, while $29 \%$ suggested that more complicated pharmacological interventions are required in women. When responses to this answer were divided by physician specialty, pain specialists unanimously expressed that pain is less tolerated, more intense, and requires more complex pharmacological intervention in women. Conversely, physiatrists were in lesser agreement about a sex difference in the experience and subsequent treatment of knee OA pain.

Eighty percent of respondents agreed that socioeconomic and cultural characteristics influence the perception of pain (Q4).

\section{Preoperative pharmacological therapy}

Eighty-six percent of respondents affirmed that the presence of comorbidities influenced their therapeutic and/or rehabilitative choices in patients with knee OA, independent of sex (Q5). Only $40 \%$ agreed that there are sex or gender differences in responses to available pain treatments in candidates for TKA (Q6). In the sub-analysis by physician specialty, $74 \%$ of physiatrists disagreed with this statement. When respondents were asked to qualify their "yes" responses to this question, most specified that they were referring to

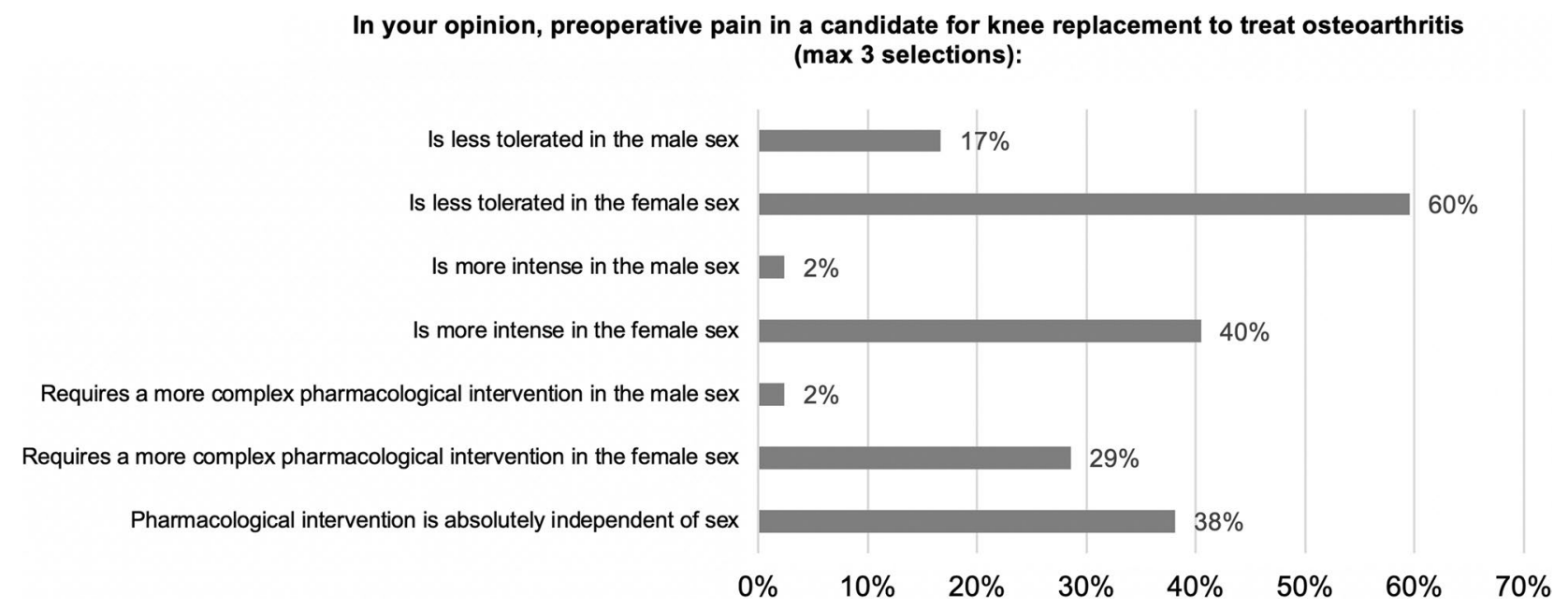

Fig. 1 Sex differences in preoperative pain severity among candidates for knee replacement. Responses to survey question 3. Respondents could provide a maximum of 3 selections 


\section{For the pharmacological management of pain in patients with knee osteoarthritis, I primarily use (max 3 selections):}

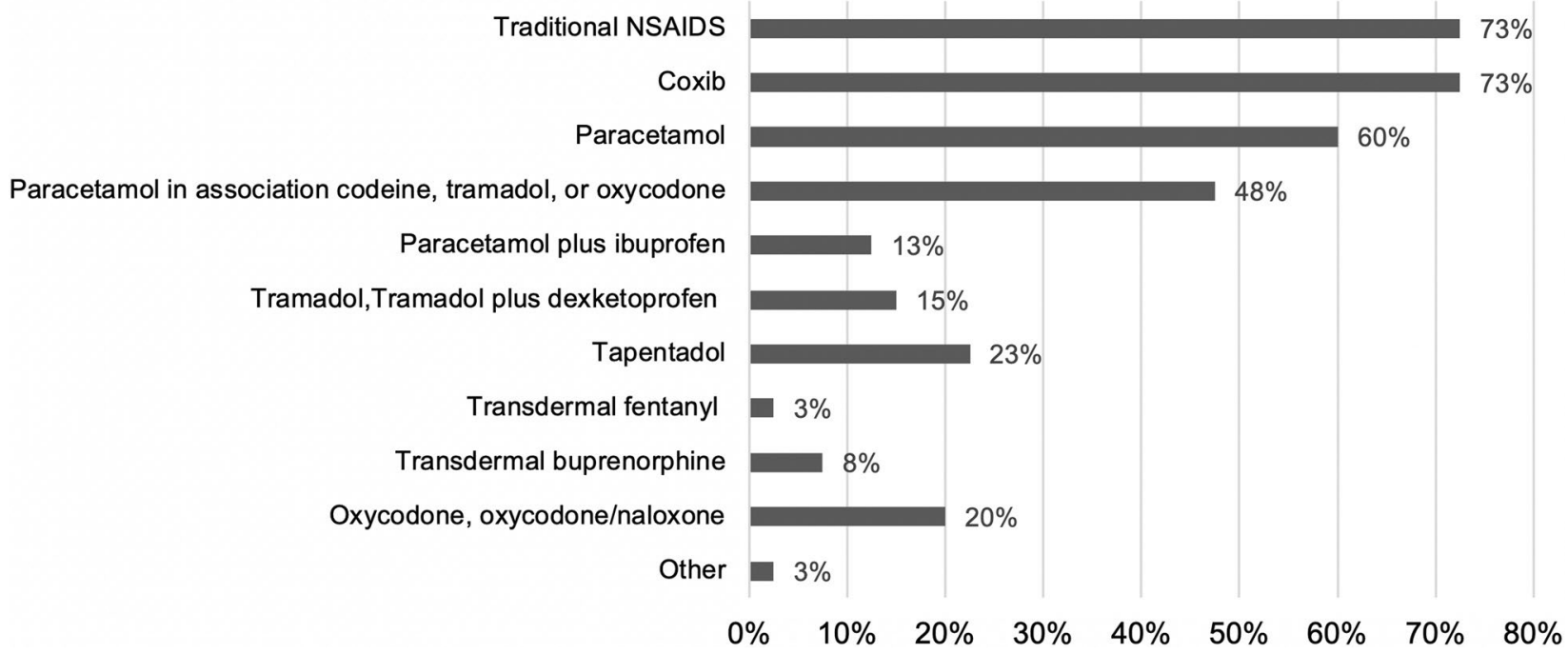

Fig. 2 Pharmacological management of pain in knee OA. Responses to survey question 8. Respondents could provide a maximum of 3 selections

non-steroidal anti-inflammatory drugs (NSAIDs), opioids, and generally lower efficacy of analgesics among women compared to men. Conversely, Ninety\% of respondents disagreed that the prescription of pharmacological treatment is guided by gender-specific indications (Q7).

With regard to the pharmacological agents prescribed by respondents to treat pain related to knee arthritis, NSAIDs and Coxib were most commonly used by $73 \%$ of respondents, followed by paracetamol (60\%) and opioid-paracetamol combinations (48\%; Q8, Fig. 2). Noteworthy, pain therapists were more likely to use tramadol alone or in combination (50\%), whereas orthopedic specialists and physiatrists primarily used NSAIDs, Coxib, and paracetamol. The most commonly prescribed oral analgesics (excluding opioids) were Coxib (83\%), diclofenac (65\%), and paracetamol (55\%; Q9). For opioids, $41 \%$ only prescribed opioids in patients with knee OA when pain was refractory to other treatments and $18 \%$ reserved opioids for moderate to severe knee pain (Q10). When responses were analyzed by physician specialty, pain therapists tended to use opioids for moderate to severe knee pain whereas physiatrists and to some extent orthopedic specialists reserved opioids for cases of refractory pain.

Finally, $45 \%$ of respondents agreed or totally agreed that in the preoperative phase, sex and gender-related differences influence responses to pharmacological treatment for pain management among patients with knee OA (Q11). Only pain specialists reflected higher agreement with the presence of a sex or gender difference, whereas physiatrists disagreed.

\section{Postoperative outcomes}

Eighty-five percent of respondents agreed that early and correct management of pain and inflammation in the perioperative phase can positively influence the final outcome of TKA independent of sex (Q12), while 13\% expressed that this was especially true among female patients. In contrast, $61 \%$ agreed that, among patients with similar clinical, functional, radiographic, and psycho-emotive characteristics, gender can significantly influence clinical outcomes of knee replacement (Q13). When responses were divided by physician specialty, a gender difference was most heavily supported by pain specialists $(88 \%)$ and to some degree orthopedics $(65 \%)$. Forty-seven percent of respondents agreed that chronic knee pain in the preoperative period (>18 months) predicts pain around the prosthesis independent of sex; $35 \%$ disagreed with the statement and $18 \%$ agreed especially in female patients (Q14). Finally, 57\% agreed that the psycho-emotional state of TKA candidates can significantly influence postoperative outcomes independent of sex (Q15). Thirty-seven percent asserted that this was especially true in female patients. When responses were divided by physician specialty, pain therapists, and orthopedic specialists were most likely to support a sex difference (Fig. 3).

\section{Rehabilitation strategy}

Sixty-two percent of respondents agreed that there were possible sex differences in the clinical and functional results of 
The psycho-emotional state of a candidate for knee replacement can significantly influence the postoperative outcome:
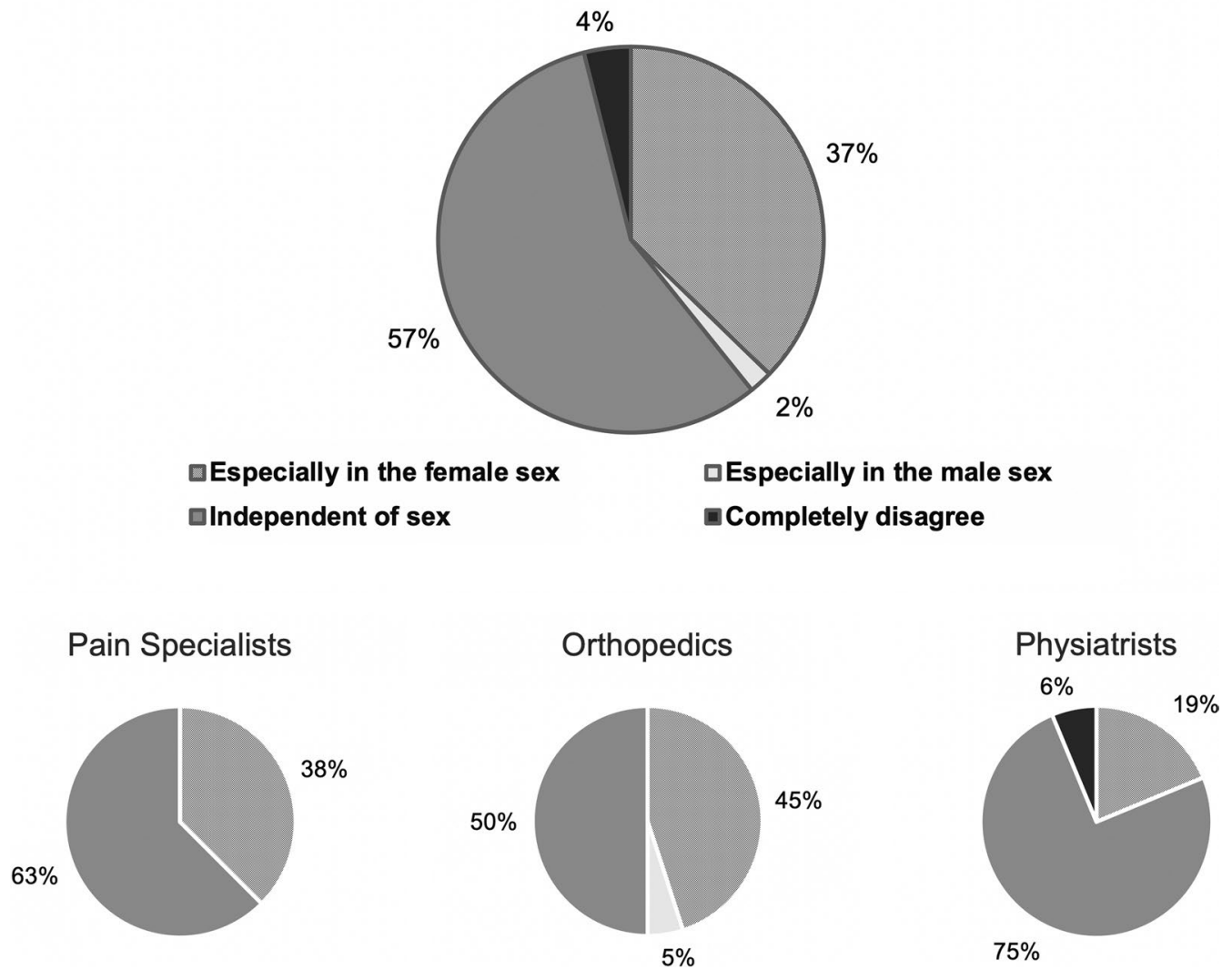

Fig. 3 Psycho-emotional state and postoperative outcomes after TKA. Responses to survey question 15 for the overall group and by physician specialty

postoperative rehabilitation after knee replacement (Q16). Yet, $76 \%$ disagreed that postsurgical treatment and rehabilitation should be guided by gender-specific indications (Q17). Respondents who agreed with this statement specified compliance as an important gender-specific difference, as well as level of pain and muscle condition or bone health prior to surgery. In a question administered only to physiatrists, 73\% disagreed that gender-specific "analgesic" physiotherapy techniques are useful for postoperative rehabilitation $(\mathrm{Q} 18)$.

\section{Postoperative patient satisfaction}

Fifty percent of respondents agreed that dissatisfaction after total knee replacement occurs independent of sex, whereas the other half qualified that dissatisfaction is more frequent in female patients (Q19). When divided by physician specialty, pain therapists, and orthopedic specialists were more likely to indicate a female predilection whereas physiatrists overwhelmingly asserted that treatment dissatisfaction is independent of sex $(87 \%)$.
Finally, $74 \%$ of respondents agreed that current literature regarding TKA is potentially biased by the use of scales that are not validated for gender-specific indications (Q20).

\section{Discussion}

The present survey of healthcare professionals indicated that physicians who treat candidates for TKA, especially pain specialists and orthopedic specialists, perceive significant sex and gender differences in disease presentation, symptom management, and treatment responses. Although these perspectives are partly supported by available literature, the survey responses emphasized a need to focus more clearly on what sex and gender differences truly mean for the clinical management of knee OA. The absence of measures and treatments that take into consideration gender-specific indications could represent a missed opportunity for improved patient care.

The interaction of pain and gender/sex is an emerging and extremely complex research topic [28-30]. In general, 
the drivers of sex differences in musculoskeletal pain can be divided into 3 categories: (1) differences in pain sensitivity and reporting, (2) differences in socioeconomic and psychological factors, and (3) sex-linked predisposing biological factors. First, literature data indicate that men and women have different responses to pain, where women are more variable in their responses, have increased pain sensitivity and have a predisposition to a larger number of painful disorders relative to men [31]. Women are also more likely to report pain than men and are, therefore, more frequently perceived as "emotional" or "overreactive", a stereotype that leads physicians to mistrust or disregard complaints of pain [32]. The interaction of higher pain sensitivity and reporting in women and physician mistrust is an important factor that may in part underlie observations that women often arrive later than men to surgery for knee OA. It is also noteworthy that women report higher postoperative pain [33], which in itself may be related to an interplay of higher pain sensitivity and late presentation for surgery with higher disease severity and disability [21,22]. Second, and also pertinent to the later observation, women are subject to different social pressures and accordingly develop divergent psychological coping mechanisms compared to men. In some cultural contexts, many women retain traditional roles in the household (e.g., cleaning, cooking, caring for sick family members) and may delay seeking care or interventions that interfere with these roles for a prolonged period of time. Women are also less likely to have a caregiver at home and instead are often the primary caregivers for family members with disability [34]. With regard to psychological coping strategies for pain, a study of 72 men and 96 women with knee OA found that higher pain and physical disability in women were mediated by catastrophizing score (using the Catastrophizing Scale of the Coping Strategies Questionnaire) [25]. Another large study found that maladaptive responses including catastrophizing were associated with worse preoperative pain and function measures in candidates for TKA [35]. Thirdly, sexlinked predisposing factors for knee OA in women include stronger predictive value of risk factors such as obesity [14], differences in cartilage composition and walking mechanics compared to men [36, 37]. differences in knee kinematics during gait both before and after TKA compared to men [38, 39], a higher annual rate of tibial and patellar cartilage loss than men [40,41], and the presence of estrogen receptors in human articular cartilage [42]. The loss of estrogen during menopause is associated with a decrease in life-long painful conditions such as headache and migraine, but can be associated with additional painful conditions such as osteoporosis and joint inflammation [43]. In summary, it is likely that perceptions of lower pain tolerance and higher pain intensity among women reported by healthcare professionals in our survey reflect a plethora of factors contributing to a true clinical difference between men and women that merits further definition in a context of knee OA.

Another important opinion expressed by physicians and especially pain specialists in our survey was that women may require a different (for example, more personalized) pharmacological approach and/or experience less efficacy of pharmacological pain treatment or poorer outcomes of TKA compared to men. Sex differences in analgesic responses have been reported wherein female sex and gender tend to be associated with lesser analgesia [33, 44]. Women are also prescribed and consume more medications than men [31]. Considering this evidence, a potentially useful approach in a context of knee OA is multimodal analgesia involving classes of analgesics that interact additively or synergistically [45-47]. This approach is historically associated with shorter hospitalization time, better recovery, and reduced healthcare costs [48]. The use of weak opioids with other agents such as NSAIDs, Coxib, or paracetamol can have an opioid-sparing effect and limit adverse events that may complicate the management of knee OA [49]. In contrast, the influence of gender or sex on TKA outcomes is less clear: although female sex was an independent risk factor for nonhome discharge in one study [27], another study found no differences in the outcomes of TKA between men and women in an Asian population [50]. A lack of definitive evidence for a sex or gender difference in TKA outcomes may be related to socioeconomic and cultural factors as well as the interaction of multiple factors with sex and gender as described above.

Another intriguing idea emerging from the survey was the importance of assessing pain and prescribing treatment using gender and sex-specific indications. In response to Q26, a majority of respondents agreed that the absence of measures validated for gender-specific indications is a shortcoming of available studies on knee OA. At present, there are no specific validated tools available to facilitate an approach tailored by sex/gender in knee OA or chronic pain in general. The development of such tools may aid future investigations of the influence of sex and gender on pain as well as the detection of sex differences in a clinically meaningful manner that can inform physician decision-making.

Having defined inherent sex and gender differences contributing to divergent disease presentation in $\mathrm{OA}$, it is equally important to examine biases among healthcare professionals and sex differences in these perceptions. First, it is noteworthy that a majority of respondents to our survey were male. Inherent sex or gender bias among male physicians has been systematically investigated: in interview studies, male physicians perceived men seeking medical help as brave and stoic, whereas women were described as sensitive, complaining, or hysterical [32]. To this extent, a previous study founded that prescriber characteristics significantly influenced pain treatment in men and women 
[51]. Male physicians in particular may benefit from gender bias sensitivity training, and the effects of such training should be investigated in future research efforts to improve the objectivity of healthcare surrounding knee OA. Second, our survey findings revealed distinct impressions about sex and gender differences in knee OA across physician specialties. Physiatrists were less likely to affirm sex and gender differences in a context of knee OA than pain specialists or orthopedic specialists. This may be in part related to the fact that pain specialists and orthopedic specialists were more likely to be male in our survey and partly related to the phase in which physicians encounter patients with knee OA. Pain specialists and orthopedic specialists are likely to see patients at peak disease and pain severity, when wellestablished sex differences in OA presentation and pain are more evident (e.g., a tendency of women present at a later stage of disease with higher peak pain that is more refractory to pharmacological treatment). Physiatrists who treat patients in the rehabilitative phase are probably witnessing a different kind of pain (i.e., pain related to physical rehabilitation) that may not be subject to the same influences of sex or gender or may be similarly intense between the two sexes. It would be useful to clarify possible sex or gender differences in pain during the rehabilitative phase after knee replacement.

The present study had a few limitations. First, the nature of this study as a survey best informs physician perceptions and not necessarily the actual nature of sex differences in knee OA, although many trends observed in our study are supported by available literature. Second, as aforementioned, a majority of respondents were male, consistent with the demographic characteristics of the included physician specialties (especially orthopedic specialists, who are more likely to be male) in Italy. Future investigations may consider balancing the sex of survey participants to better inform perceived sex and gender differences in healthcare settings. Third, this survey was restricted to physicians in Italy and may reflect some cultural bias. A recent decree (law 3/2018 article 3) calls for the integration of gender medicine into the practices of the Italian National Health Service, but a lack of education and training among physicians nationwide represents an important obstacle to its successful implementation. Finally, it should be noted that, while sex and gender differences in knee OA have important bearing on its clinical management, they represent only one dimension of a multifactorial influence on outcome comprised of socioeconomic status, comorbidities, and other variables. Studies should consider whether disparities are due to sex or gender differences in disease incidence, disease expression or severity, or additional factors by examining a variety of inequity types. Further research is needed to better inform the influences of these factors individually and together on the management and outcomes of knee OA.

\section{Conclusions}

In conclusion, the results of our survey demonstrate that healthcare professionals perceive multiple sex and genderrelated differences in patients with knee OA, especially in the pre and perioperative phases of TKA. The exact nature and significance of these differences between men and women should be better investigated with regard to differences in disease severity; clinical factors at initial presentation; physical, biochemical, and psycho-emotive characteristics; and socioeconomic as well as cultural factors. At the same time, it is necessary to evaluate bias in physician perceptions about sex and gender. In Italy [52] and worldwide, the study of gender differences and the development of gender-based medicine are considered relevant to the advancement of life sciences. Sex and gender analysis can help reveal and assess inequality and can improve actions to create institutional change to ensure greater equity Future efforts are necessary to synthesize these observations into clinically translatable sex- or gender-specific indications to improve care in patients with knee OA.

\section{Appendix (Questions)}

1. Express your agreement or disagreement with the following statement: "At the first specialist visit for knee arthritis, male patients present with more severe symptomology than female patients."
a. Completely disagree
b. Disagree
c. Agree
d. Totally agree

2. Express your agreement or disagreement with the following statement: "In patients with equivalent clinical and functional characteristics, female patients with knee arthritis present with a poorer subjective perception of functional limitation than male patients."
a. Completely disagree
b. Disagree
c. Agree
d. Totally agree 
3. In your opinion, preoperative pain in a candidate for knee replacement to treat osteoarthritis: (indicate a maximum of 3 options below)

a. The intensity is absolutely independent of sex

b. Is more intense in the female sex

c. Is more intense in the male sex

d. Is less tolerated in the female sex

e. Is less tolerated in the male sex

f. Pharmacological intervention is absolutely independent of sex

g. Requires a more complex pharmacological intervention in the female sex

h. Requires a more complex pharmacological intervention in the male sex

4. Express your agreement or disagreement with the following statement: "The socioeconomic and cultural characteristics of a patient influence their perception of pain."
a. Completely disagree
b. Disagree
c. Agree
d. Totally agree

5. Does the presence of comorbidities in patients with osteoarthritis of the knee influence your choice of therapy and/or rehabilitation?
a. No
b. Yes, especially for the female sex
c. Yes, independent of sex
d. Yes, especially for the male sex

6. Do sex or gender differences influence the response to available pain treatments in candidates for knee replacement? (If yes, specify.)
a. Yes
b. No

7. Is the prescription of pharmacological treatment guided by gender-specific indications? (If yes, specify.)
a. Yes
b. No

8. For the pharmacological management of pain in patients with knee osteoarthritis, I primarily use: (indicate a maximum of 3 options below)

a. Oxycodone, oxycodone/naloxone b. Transdermal buprenorphine

c. Transdermal fentanyl

d. Tapentadol

e. Tramodol plus dexketoprofen

f. Tramadol

g. Paracetamol plus ibuprofen

h. Paracetamol in association with opioids (codeine, tramadol, or oxycodone)

i. Paracetamol

j. Coxib

k. Traditional NSAIDs

1. Other

9. Regarding oral analgesics (excluding opioids), patients with knee osteoarthritis most frequently use: (indicate a maximum of three options below)
a. Diclofenac
b. Paracetamol plus NSAIDs
c. Paracetamol
d. Ibuprofen
e. Coxib
f. Naproxen
g. Other

10. I prescribe opioids for pain management in patients with knee osteoarthritis:

a. I never prescribe opioids in these patients

b. I only prescribe weak opioids as needed

c. In patients with knee osteoarthritis and moderate to severe pain

d. Only during the preoperative period to favor early rehabilitation

e. Only for short periods in all patients

f. Only in the presence of pain refractory to other treatments

g. Only during the perioperative phase (24-72 h)

h. Other

11. Express your agreement or disagreement with the following statement: "In the preoperative phase, sex or gender differences influence the response to pharmacological treatment for pain management in patients with knee osteoarthritis."
a. Completely disagree
b. Disagree
c. Agree
d. Totally agree 
12. The early and correct perioperative management of pain and inflammation can positively influence the final outcome:
a. Especially in female patients
b. Especially in male patients
c. Independent of sex

13. Express your agreement or disagreement with the following statement: "In patients with equivalent clinical/ functional scores, psycho-emotive status, and radiographic characteristics, gender differences can significantly influence postoperative clinical outcomes."
a. Completely disagree
b. Disagree
c. Agree
d. Totally agree

14. Patients with a history of chronic knee pain preoperatively ( $>18$ months) are predisposed to pain around the prosthesis after knee replacement.
a. Especially in female patients
b. Especially in male patients
c. Independent of sex
d. I completely disagree with the statement

15. The psycho-emotional state of a candidate for knee replacement can significantly influence the postoperative outcome.
a. Especially in female patients
b. Especially in male patients
c. Independent of sex
d. I completely disagree with the statement

16. Express your agreement or disagreement with the following statement: "The clinical and functional results of postoperative rehabilitation after knee replacement can vary by sex."*
a. Completely disagree
b. Disagree
c. Agree
d. Totally agree

*Question not administered to pain therapists

17. In patients with knee replacement, do you believe that the choice of postsurgical rehabilitative treatment should be guided by gender-specific indications? (If yes, specify.)

a. Yes
b. No

18. Do you find gender-specific analgesic physiotherapy techniques to be useful during the postoperative rehabilitation phase? (If yes, specify.)*
a. Yes
b. No

*Question not administered to pain therapists or orthopedics.

19. Dissatisfaction after complete knee replacement:
a. Is more frequent in male patients
b. Is more frequent in female patients
c. Is independent of sex

20. Express your agreement or disagreement with the following statement: "The various positive and negative results of knee replacement published in the literature could be biased by the use of scales that are not validated for gender-specific indications.
a. Completely disagree
b. Disagree
c. Agree
d. Totally agree
e. I don't know

Acknowledgements Authors thank Claudia Laterza, M.D. of Sanitanova S.r.l. and Ashley Symons, Ph.D. for draft preparation and editorial assistance

Author contributions All authors participated in this research as members of the scientific board and were, therefore, involved in all aspects of the study including design of the survey, analysis and discussion of the results, and critical review of the final manuscript.

Funding This research and related manuscript writing and editing activities were funded by an unconditional grant from Angelini S.p.A.

Availability of data and materials The datasets used and/or analyzed during the current study are available from the corresponding author on reasonable request.

\section{Declarations}

Coflict of interest G. Varrassi is a consultant for Abbott, Angelini, Dompé, Grünenthal, Malesci, Menarini, Molteni, Pfizer, Shonogi, and Takeda. He has received research grants from Dompé, the Fondazione Internazionale Maugeri, and the Consiglio Nazionale per la Ricerca. He also serves on the Editorial Board of several scientific journals and is the Editor and Chief of Pain and Therapy. The other authors declare that they have no competing interests. 
Open Access This article is licensed under a Creative Commons Attribution 4.0 International License, which permits use, sharing, adaptation, distribution and reproduction in any medium or format, as long as you give appropriate credit to the original author(s) and the source, provide a link to the Creative Commons licence, and indicate if changes were made. The images or other third party material in this article are included in the article's Creative Commons licence, unless indicated otherwise in a credit line to the material. If material is not included in the article's Creative Commons licence and your intended use is not permitted by statutory regulation or exceeds the permitted use, you will need to obtain permission directly from the copyright holder. To view a copy of this licence, visit http://creativecommons.org/licenses/by/4.0/.

\section{References}

1. Vos T, Flaxman AD, Naghavi M et al (2012) Years lived with disability (YLDs) for 1160 sequelae of 289 diseases and injuries 1990-2010: a systematic analysis for the global burden of disease study 2010. Lancet (London, England) 380:2163-2196

2. Safiri S, Kolahi A-A, Smith E et al (2020) Global, regional and national burden of osteoarthritis 1990-2017: a systematic analysis of the Global Burden of Disease Study 2017. Ann Rheum Dis 79:819-828

3. Mannoni A, Briganti MP, Di Bari M, Ferrucci L, Costanzo S, Serni U, Masotti G, Marchionni N (2003) Epidemiological profile of symptomatic osteoarthritis in older adults: a population based study in Dicomano, Italy. Ann Rheum Dis 62:576-578

4. Heidari B (2011) Knee osteoarthritis prevalence, risk factors, pathogenesis and features: Part I. Casp J Intern Med 2:205-212

5. Marmot M, Friel S, Bell R, Houweling TAJ, Taylor S, Commission on Social Determinants of Health, (2008) Closing the gap in a generation: health equity through action on the social determinants of health. Lancet (London, England) 372:1661-1669

6. World health Organization (2011) Gender mainstreaming for health managers: a practical approach. https://apps.who.int/iris/ handle/10665/44516. Accessed 9 June 2021

7. World Health Organization (2015): Integrating equity, gender, human rights and social determinants into the work of WHO Roadmap for Action 2014-2019 . https://www.who.int/genderequity-rights/knowledge/web-roadmap.pdf?ua=1. Accessed 9 June 2021

8. Basques BA, Bell JA, Fillingham YA, Khan JM, Della Valle CJ (2019) Gender differences for hip and knee arthroplasty: complications and healthcare utilization. J Arthroplasty 34:1593-1597. e1

9. Devasenapathy N, Malhotra R, Mittal K, Garg B, Kumar V, Zodpey S, Dogra H, Maddison R, Belavy DL (2020) Higher disability in women than men scheduled for total knee arthroplasty for degenerative osteoarthritis: a cross-sectional analysis from India. ACR open Rheumatol 2:309-319

10. Gallo J, Kriegova E, Kudelka M, Lostak J, Radvansky M (2020) Corrigendum to "gender differences in contribution of smoking, low physical activity, and high bmi to increased risk of early reoperation after TKA" [The Journal of Arthroplasty 35 (2020) 1545-1557]. J Arthroplasty 35:2694

11. Lo CWT, Tsang WWN, Yan CH, Lord SR, Hill KD, Wong AYL (2019) Risk factors for falls in patients with total hip arthroplasty and total knee arthroplasty: a systematic review and meta-analysis. Osteoarthr Cartil 27:979-993

12. Oh C, Gold H, Slover J (2020) Diagnosis of depression and other patient factors impacts length of stay after total knee arthroplasty. Arthroplast Today 6:77-80
13. Uccheddu D, Gauthier AH, Steverink N, Emery T (2019) Gender and socioeconomic inequalities in health at older ages across different european welfare clusters: evidence from SHARE data, 2004-2015. Eur Sociol Rev 35:346-362

14. Nicolella DP, O'Connor MI, Enoka RM et al (2012) Mechanical contributors to sex differences in idiopathic knee osteoarthritis. Biol Sex Differ 3:28

15. O'Connor MI (2006) Osteoarthritis of the hip and knee: sex and gender differences. Orthop Clin North Am 37:559-568

16. Srikanth VK, Fryer JL, Zhai G, Winzenberg TM, Hosmer D, Jones $\mathrm{G}$ (2005) A meta-analysis of sex differences prevalence, incidence and severity of osteoarthritis. Osteoarthr Cartil 13:769-781

17. Kluzek S, Sanchez-Santos MT, Leyland KM, Judge A, Spector TD, Hart D, Cooper C, Newton J, Arden NK (2016) Painful knee but not hand osteoarthritis is an independent predictor of mortality over 23 years follow-up of a population-based cohort of middleaged women. Ann Rheum Dis 75:1749-1756

18. Hawker G, Wright J, Coyte P, Paul J, Dittus R, Croxford R, Katz B, Bombardier C, Heck D, Freund D (1998) Health-related quality of life after knee replacement. J Bone Joint Surg Am 80:163-173

19. Liang MH, Cullen KE, Larson MG, Thompson MS, Schwartz JA, Fossel AH, Roberts WN, Sledge CB (1986) Cost-effectiveness of total joint arthroplasty in osteoarthritis. Arthritis Rheum 29:937-943

20. Piscitelli P, Iolascon G, Di Tanna G et al (2012) Socioeconomic burden of total joint arthroplasty for symptomatic hip and knee osteoarthritis in the Italian population: a 5-year analysis based on hospitalization records. Arthritis Care Res (Hoboken) 64:1320-1327

21. Hawker GA, Wright JG, Coyte PC, Williams JI, Harvey B, Glazier R, Badley EM (2000) Differences between men and women in the rate of use of hip and knee arthroplasty. N Engl J Med 342:1016-1022

22. Parsley BS, Bertolusso R, Harrington M, Brekke A, Noble PC (2010) Influence of gender on age of treatment with TKA and functional outcome. Clin Orthop Relat Res 468:1759-1764

23. Bawa HS, Weick JW, Dirschl DR (2016) Gender disparities in osteoarthritis-related health care utilization before total knee arthroplasty. J Arthroplasty 31:2115-2118.e1

24. Templeton KJ (2020) Sex and gender issues in pain management. J Bone Joint Surg Am 102(Suppl):32-35

25. Keefe FJ, Lefebvre JC, Egert JR, Affleck G, Sullivan MJ, Caldwell DS (2000) The relationship of gender to pain, pain behavior, and disability in osteoarthritis patients: the role of catastrophizing. Pain 87:325-334

26. Bartley EJ, Fillingim RB (2013) Sex differences in pain: a brief review of clinical and experimental findings. Br J Anaesth 111:52-58

27. Robinson J, Shin JI, Dowdell JE, Moucha CS, Chen DD (2017) Impact of gender on 30-day complications after primary total joint arthroplasty. J Arthroplasty 32:2370-2374

28. Hines $M(2020)$ Neuroscience and sex/gender: looking back and forward. J Neurosci 40:37-43

29. Iolascon, (2017) Early osteoarthritis: how to define, diagnose, and manage. A systematic review Eur Geriatric Med 8:383-396

30. Mauvais-Jarvis F, Bairey Merz N, Barnes PJ et al (2020) Sex and gender: modifiers of health, disease, and medicine. Lancet 396:565-582

31. Pieretti S, Di Giannuario A, Di Giovannandrea R, Marzoli F, Piccaro G, Minosi P, Aloisi AM (2016) Gender differences in pain and its relief. Ann Ist Super Sanita 52:184-189

32. Samulowitz A, Gremyr I, Eriksson E, Hensing G (2018) "Brave Men" and "emotional women": a theory-guided literature review on gender bias in health care and gendered norms towards patients with chronic pain. Pain Res Manag 2018:6358624 
33. Fillingim RB (2002) Sex differences in analgesic responses: evidence from experimental pain models. Eur J Anaesthesiol Suppl 26:16-24

34. National Center on Caregiving at Family Caregiver Alliance (2015). Women and Caregiving: Facts and Figures. https://www. caregiver.org/resource/women-and-caregiving-facts-and-figures/. Accessed 9 June 20121

35. Riddle DL, Jensen MP, Ang D, Slover J, Perera R, Dumenci L (2018) Do pain coping and pain beliefs associate with outcome measures before knee arthroplasty in patients who catastrophize about pain? A cross-sectional analysis from a randomized clinical trial. Clin Orthop Relat Res 476:778-786

36. Blagojevic M, Jinks C, Jeffery A, Jordan KP (2010) Risk factors for onset of osteoarthritis of the knee in older adults: a systematic review and meta-analysis. Osteoarthr Cartil 18:24-33

37. Kumar D, Souza RB, Subburaj K et al (2015) Are there sex differences in knee cartilage composition and walking mechanics in healthy and osteoarthritis populations? Clin Orthop Relat Res 473:2548-2558

38. Astephen Wilson JL, Dunbar MJ, Hubley-Kozey CL (2015) Knee joint biomechanics and neuromuscular control during gait before and after total knee arthroplasty are sex-specific. J Arthroplasty 30:118-125

39. Leszko F, Hovinga KR, Lerner AL, Komistek RD, Mahfouz MR (2011) In vivo normal knee kinematics: is ethnicity or gender an influencing factor? Clin Orthop Relat Res 469:95-106

40. Brennan SL, Cicuttini FM, Shortreed S, Forbes A, Jones G, Stuckey SL, Wluka AE (2010) Women lose patella cartilage at a faster rate than men: a 4.5 -year cohort study of subjects with knee OA. Maturitas 67:270-274

41. Hanna FS, Teichtahl AJ, Wluka AE, Wang Y, Urquhart DM, English DR, Giles GG, Cicuttini FM (2009) Women have increased rates of cartilage loss and progression of cartilage defects at the knee than men: a gender study of adults without clinical knee osteoarthritis. Menopause 16:666-670

42. Ushiyama T, Ueyama H, Inoue K, Ohkubo I, Hukuda S (1999) Expression of genes for estrogen receptors alpha and beta in human articular chondrocytes. Osteoarthr Cartil 7:560-566

43. Meriggiola MC, Nanni M, Bachiocco V, Vodo S, Aloisi AM (2012) Menopause affects pain depending on pain type and characteristics. Menopause 19:517-523
44. Packiasabapathy S, Sadhasivam S (2018) Gender, genetics, and analgesia: understanding the differences in response to pain relief. J Pain Res 11:2729-2739

45. Iolascon G, Ruggiero C, Fiore P, Mauro GL, Moretti B, Tarantino U (2020) Multidisciplinary integrated approach for older adults with symptomatic osteoarthritis: SIMFER and SI-GUIDA joint position statement. Eur J Phys Rehabil Med 56:112-119

46. Migliore A, Gigliucci G, Alekseeva L et al (2019) Treat-to-target strategy for knee osteoarthritis. International technical expert panel consensus and good clinical practice statements. Ther Adv Musculoskelet Dis 11:1759720X19893800

47. Migliore A, Paoletta M, Moretti A, Liguori S, Iolascon G (2020) The perspectives of intra-articular therapy in the management of osteoarthritis. Expert Opin Drug Deliv 17:1213-1226

48. Castillo RC, Raja SN, Frey KP et al (2017) Improving pain management and long-term outcomes following high-energy orthopaedic trauma (pain study). J Orthop Trauma 31(Suppl 1):S71-S77

49. Webb CAJ, Mariano ER (2015) Best multimodal analgesic protocol for total knee arthroplasty. Pain Manag 5:185-196

50. Gen LY, Bin Abd Razak HR, Chi CH, Chye TH (2015) No gender-based differences in outcomes after conventional total knee arthroplasty in Asians. J Arthroplasty 30:1548-1550

51. Leresche L (2011) Defining gender disparities in pain management. Clin Orthop Relat Res 469:1871-1877

52. Art 3 Legge 3/2018: Delega al Governo in materia di sperimentazione clinica di medicinali nonchè disposizioni per il riordino delle professioni sanitarie e per la dirigenza sanitaria del Ministero della salute. (GU Serie Generale n.25 del 31-01-2018) https:// www.gazzettaufficiale.it/eli/id/2018/1/31/18G00019/sg. Accessed 9 June 2021

Publisher's Note Springer Nature remains neutral with regard to jurisdictional claims in published maps and institutional affiliations. 